\title{
LIGAÇÕES ENTRE DIAGNÓSTICOS, RESULTADOS E INTERVENÇÕES DE ENFERMAGEM PARA PACIENTES NO PERÍODO TRANSOPERATÓRIO
}

\author{
Links between diagnostics, results and nursing \\ interventions for patients in the transoperative period \\ Conexiones entre diagnósticos, resultados e intervenciones de \\ enfermería para pacientes em el período transoperatorio
Djulia Camila Berwanger', Fabiana Gonçalves de Oliveira Azevedo Matos ${ }^{2 *}$, Débora Cristina Ignácio Alves ${ }^{3}$, João Lucas Campos de Oliveira ${ }^{4}$

RESUMO: Objetivos: Construir e validar as ligações entre os principais diagnósticos, resultados e intervenções de enfermagem para pacientes no período transoperatório. Método: Estudo metodológico desenvolvido em três etapas: revisão integrativa da literatura, para identificar os diagnósticos, resultados e intervenções de enfermagem mais comuns no período transoperatório; construção de material teórico com base na pesquisa prévia e na experiência das autoras, contendo a ligação entre os principais diagnósticos, resultados e intervenções; e submissão do material construído à avaliação de conteúdo por especialistas nacionalmente reconhecidos pela atuação na referida área de conhecimento. A investigação teve aprovação do Comitê de Ética em Pesquisa. Resultados: A versão final do material construído ficou composta da ligação de 27 diagnósticos, 25 resultados, 26 intervenções e 141 atividades de enfermagem. Conclusão: Acredita-se que os resultados do estudo possam ser utilizados para fundamentar a prática clínica de enfermeiros que atuam em centro cirúrgico. Conhecer os elementos mais comuns do processo de cuidar no período transoperatório otimiza a assistência prestada, conferindo mais autonomia e segurança para a tomada de decisão.

Palavras-chave: Enfermagem de centro cirúrgico. Enfermagem perioperatória. Processo de enfermagem. Diagnóstico de enfermagem. Cuidados de enfermagem.

ABSTRACT: Objectives: To build and to validate the links between the main diagnoses, results and nursing interventions for the patients in the transoperative period. Method: Methodological study developed in three stages: integrative revision of the literature, to identify the most common diagnoses, results and nursing interventions in the transoperative period; construction of theoretical material, based on the prior research and experience of the authors, containing the link between the main diagnoses, results and interventions; and submission of the material built to the evaluation of content by experts nationally recognized by the performance in the said area of knowledge. The investigation was approved by the Ethics Committee on Research. Results: The final version of the constructed material was composed of the link of 27 diagnoses, 25 results, 26 interventions and 141 nursing activities. Conclusion: It is believed that the results of the study can be used to substantiate the clinical practice of nurses in the surgical center. Knowing the most common elements of the process of caring in the transoperative period optimizes the assistance provided, giving more autonomy and security for decision making.

Keywords: Operating room nursing. Perioperative nursing. Nursing process. Nursing diagnosis. Nursing care.

'Enfermeira; residente de Enfermagem em Clínica Médica e Cirúrgica do Hospital Universitário do Oeste do Paraná (HUOP) - Cascavel (PR), Brasil.

Enfermeira; doutora em Ciências pela Escola de Enfermagem da Universidade de São Paulo (USP); docente da Universidade Estadual do Oeste do Paraná (Unioeste) - Cascavel (PR), Brasil.

${ }^{3}$ Enfermeira; doutora em Ciências pela Escola de Enfermagem da USP; docente da Unioeste - Cascavel (PR).

«Enfermeiro; doutor em Enfermagem; professor adjunto da Faculdade de Enfermagem da Universidade Federal de Mato Grosso (UFMT) - Cuiabá (MT), Brasil.

*Autor Correspondente: fabianamatos@hotmail.com

Recebido: 28/02/2018 - Aprovado: 20/05/2018

DOI: $10.5327 / Z 1414-4425201800040002$ 
RESUMEN: Objetivos: Construir y legitimar las conexiones entre los principales diagnósticos, resultados e intervenciones de enfermería para pacientes en el período transoperatorio. Método: Estudio metodológico, desarrollado en tres etapas: revisión integrativa de la literatura, para identificar los diagnósticos, resultados e intervenciones de enfermería más comunes en el período transoperatorio; construcción de material teórico basado en la investigación previa y en la experiencia de las autoras, conteniendo la conexión entre los diagnósticos principales, resultados e intervenciones; y sujeción del material construído a la evaluación del contenido por expertos nacionalmente reconocidos por actuar en la referida área de conocimento. La investigación tuvo la aprobación del Comité de Ética en Investigación. Resultados: La versión final del material construído quedó compuesta por la conexión de 27 diagnósticos, 25 resultados, 26 intervenções y 141 atividades de enfermería. Conclusión: Se cree que los resultados del estudio podrían utilizarse para justificar la práctica clínica de enfermeros que actúan en el quirófano. Conocer los elementos más comunes del proceso de cuidar en el período transoperatorio, optimiza la asistencia prestada, otorgando más autonomía y seguridad para la toma de decisión.

Palabras clave: Enfermería de quirófano. Enfermería perioperatoria. Proceso de enfermería. Diagnóstico de enfermería. Atención de enfermería.

\section{INTRODUÇÃO}

A enfermagem, por meio do conhecimento técnico-científico adquirido, utiliza metodologias de cuidado sistemáticas para organizar e prestar assistência haja vista as necessidades de cuidados identificados. Assim, o emprego do processo de enfermagem $(\mathrm{PE})$ na prática clínica do enfermeiro permite o uso de Sistemas de Linguagens Padronizadas (SLP) dos elementos do processo de cuidar, compreendido por diagnósticos, resultados e intervenções de enfermagem ${ }^{1}$. Quando padronizados, esses elementos funcionam como organizadores e aceleradores do raciocínio clínico de enfermagem ${ }^{2}$, pois clarificam conceitos, apontam indicadores clínicos, descrevem as ações e auxiliam na mensuração dos resultados ${ }^{1}$.

Com o intuito de padronizar a redação dos diagnósticos de enfermagem, na década de 1970 foi criada a North American Nursing Diagnosis Association (NANDA). Em 2002, a referida organização passou a ser denominada de NANDA Internacional (NANDA-I), com a finalidade de refletir o cenário mundial que representava ${ }^{3}$. Da mesma forma, objetivando padronizar as ações de enfermagem prescritas pelos enfermeiros e os resultados de enfermagem a serem alcançados, foram criadas outras classificações de enfermagem, como a Classificação das Intervenções de Enfermagem (NIC) ${ }^{4}$ e a Classificação dos Resultados de Enfermagem (NOC) $)^{5}$, algumas das classificações mais difundidas internacionalmente.

Tais classificações de linguagens padronizadas compreendem um aparato de trabalho sistematizado e robusto para ser utilizado em conjunto. "Estabelecer ligações entre essas três linguagens ajuda clínicos e estudantes na escolha dos resultados e intervenções mais apropriados aos diagnósticos de enfermagem de seus clientes"6.

Para orientar os cuidados de enfermagem realizados nos períodos pré, trans e pós-operatório, foi desenvolvido, em meados de 1980, um modelo chamado de Sistematização da Assistência de Enfermagem Perioperatória $(\mathrm{SAEP})^{7}$, cuja finalidade é atender aos pacientes cirúrgicos e seus familiares de maneira segura, integral, individualizada e contínua ${ }^{8,9}$. No entanto, por conta da dinâmica complexa do centro cirúrgico (CC) e da necessidade do cumprimento de inúmeras atividades assistenciais e gerenciais inerentes ao setor, o enfermeiro encontra dificuldades para realizar a SAEP em sua prática diária9,10.

\section{OBJETIVOS}

Construir e validar as ligações entre os principais diagnósticos, resultados e intervenções de enfermagem para o paciente no período transoperatório.

\section{MÉTODO}

Trata-se de estudo metodológico desenvolvido em três etapas distintas. Na primeira etapa, fez-se a revisão integrativa da literatura, para identificar os principais diagnósticos, resultados e intervenções de enfermagem elencados para pacientes no período transoperatório.

A revisão integrativa foi realizada em periódicos indexados nas seguintes bases eletrônicas: Medical Literature Analysis and Retrieval Sistem Online (MEDLINE), Scientific Eletronic Library Online (SciELO) e Literatura Latino-Americana e do Caribe em Ciências da Saúde (LILACS). Para o levantamento bibliográfico, foram utilizados os seguintes descritores controlados, de acordo com os parâmetros dos Descritores em Ciências da Saúde (DeCS): "Enfermagem de centro cirúrgico", "Diagnóstico de enfermagem" e "Cuidados de 
enfermagem", combinados entre si por meio dos operadores booleanos "AND" e "OR". Além disso, empregaram-se os seguintes termos livres: "Diagnóstico de enfermagem transoperatório" e "Assistência de enfermagem perioperatória".

Os critérios de inclusão dos artigos científicos foram: trabalhos publicados em português, espanhol ou inglês cujos textos completos estão disponíveis online nas bases selecionadas e sem limite de tempo de publicação, em razão da escassez de estudos sobre a temática. A amostra final foi constituída de 20 artigos científicos.

$\mathrm{Na}$ segunda etapa foi construído, pelas autoras, com base na revisão integrativa da literatura e em suas experiências clínicas em enfermagem perioperatória, um material teórico com os principais diagnósticos, resultados e intervenções de enfermagem para pacientes no período transoperatório que foram elencados conforme as classificações NANDA-I $\mathrm{I}^{3}$, $\mathrm{NOC}^{5}$, e NIC, ${ }^{4}$ respectivamente. O referido material contém a ligação entre 27 diagnósticos, 25 resultados, 26 intervenções e 137 atividades de enfermagem.

Na terceira etapa, o material construído foi submetido à validação de conteúdo por especialistas nacionalmente reconhecidos pela atuação na referida área de conhecimento. Foram considerados especialistas os enfermeiros que possuem publicação sobre diagnósticos, resultados ou intervenções de enfermagem no período perioperatório; desenvolveram, durante o curso de mestrado ou doutorado, pesquisas sobre diagnósticos, resultados e intervenções de enfermagem, enfermagem perioperatória ou enfermagem em clínica médica e cirúrgica; e tinham à época prática clínica mínima de um ano na unidade de CC, ou em clínica médica ou cirúrgica. A identificação dos enfermeiros expertos foi feita por meio de pesquisa online aos currículos da Plataforma Lattes contidos no portal do Conselho Nacional de Desenvolvimento Científico e Tecnológico (CNPq).

Após a análise dos currículos, 23 especialistas foram consultados via correio eletrônico sobre o interesse em fazer parte do estudo. Dos enfermeiros consultados, 10 aceitaram contribuir com a pesquisa; sete não concordaram, justificando problemas com a agenda de trabalho; e seis não responderam aos e-mails enviados. No entanto, dos 10 enfermeiros que afirmaram que participariam do estudo, apenas sete retornaram o instrumento preenchido no tempo estipulado para a coleta de dados.

Aos enfermeiros que aceitaram integrar o estudo, foram enviados o instrumento de coleta de dados, um guia de orientações de como preencher esse instrumento e o termo de consentimento livre e esclarecido (TCLE). A coleta de dados foi realizada de setembro a dezembro de 2017. A avaliação de especialistas objetivou verificar a pertinência (sim ou não) dos elementos do processo de cuidar à realidade vivenciada pelo paciente durante o período transoperatório.

A concordância aceitável entre os avaliadores foi determinada com base no total de especialistas participantes no estudo. Quando há participação de seis ou mais avaliadores, recomenda-se que a taxa de concordância não seja inferior a $0,78^{11}$. A análise dos dados foi feita por meio de estatística descritiva, expressa em frequência absoluta (n) e relativa (\%).

O estudo teve aprovação do Comitê de Ética em Pesquisa, $\mathrm{n}^{\circ}$ do Certificado de Apresentação para Apreciação Ética (CAAE) 70825517.5.0000.010.

\section{RESULTADOS}

A totalidade dos enfermeiros que participou do estudo como especialista $(n=7 ; 100 \%)$ era do sexo feminino, possuía titulação acadêmica em nível de doutorado e tinha, no mínimo, um artigo publicado sobre diagnósticos, resultados e/ou intervenções de enfermagem no período perioperatório.

Todos os diagnósticos $(\mathrm{n}=27,100 \%)$, resultados $(\mathrm{n}=25$, $100 \%)$ e intervenções de enfermagem $(n=26,100 \%)$ propostos alcançaram concordância adequada entre os avaliadores. Das $137(100 \%)$ atividades de enfermagem avaliadas, apenas duas $(n=2 ; 1,5 \%)$ obtiveram concordância abaixo de $80 \%$ entre os avaliadores, e elas foram excluídas do instrumento. Outras seis atividades de enfermagem foram incorporadas por sugestão dos especialistas.

Dessa forma, o material contendo as ligações entre os principais diagnósticos, resultados e intervenções para o paciente no período transoperatório ficou composto de 27 diagnósticos, 25 resultados, 26 intervenções e 141 atividades de enfermagem. O Quadro 1 traz a versão validada pelos especialistas. A apresentação dos dados de estudo no referido quadro foi feita por ordem alfabética dos diagnósticos .

\section{DISCUSSÃO}

Como o objetivo do estudo foi propor ligações entre os elementos estruturantes do cuidado de enfermagem, é importante ressaltar que todo esse processo depende da correta identificação dos diagnósticos, visto que interpretações 
Quadro 1. Ligação entre diagnósticos, resultados e intervenções de enfermagem para o período transoperatório.

\begin{tabular}{|c|c|c|}
\hline $\begin{array}{l}\text { Diagnósticos de } \\
\text { enfermagem (NANDA-I) }\end{array}$ & $\begin{array}{l}\text { Resultados de } \\
\text { enfermagem } \\
\text { (NOC) }\end{array}$ & Intervenções de enfermagem (NIC) \\
\hline \multirow{5}{*}{ Ansiedade (00146) } & \multirow{5}{*}{$\begin{array}{l}\text { Nível de ansiedade } \\
\qquad(1211) \\
\text { (Indicador: ansiedade } \\
\quad \text { verbalizada) }\end{array}$} & Redução da ansiedade (5820) \\
\hline & & Utilizar abordagem calma e tranquilizadora. \\
\hline & & Escutar atentamente. \\
\hline & & Permanecer com o paciente para promover segurança e diminuir o medo. \\
\hline & & Administrar medicamentos para reduzir a ansiedade, conforme apropriado. \\
\hline \multirow{7}{*}{$\begin{array}{l}\text { Capacidade de transferência } \\
\text { prejudicada (00090) }\end{array}$} & \multirow{7}{*}{$\begin{array}{l}\text { Desempenho na } \\
\text { transferência }(0210) \\
\text { (Indicador: transferência } \\
\text { de uma superfície para } \\
\text { a outra) }\end{array}$} & Assistência no autocuidado: transferência (1806) \\
\hline & & Selecionar técnica de transferência apropriada para o paciente. \\
\hline & & Usar mecânica corporal apropriada durante os movimentos. \\
\hline & & Manter o corpo do paciente corretamente alinhado durante os movimentos. \\
\hline & & $\begin{array}{l}\text { Avaliar o paciente no fim da transferência quanto ao alinhamento adequado } \\
\text { do corpo, à desobstrução de dispositivos, a roupas de cama sem dobras, } \\
\text { à região do corpo exposta desnecessariamente, a conforto adequado e } \\
\text { grades levantadas. }\end{array}$ \\
\hline & & Zelar pela privacidade do paciente durante a transferência. \\
\hline & & Travar as rodas da cama/maca durante a transferência do paciente. \\
\hline \multirow{4}{*}{$\begin{array}{l}\text { Comunicação verbal } \\
\text { prejudicada (00051) }\end{array}$} & \multirow{4}{*}{$\begin{array}{l}\text { Comunicação (0902) } \\
\text { (Indicador: interpretação } \\
\text { precisa de mensagens } \\
\text { recebidas) }\end{array}$} & Melhora da comunicação: déficit da fala (4976) \\
\hline & & $\begin{array}{l}\text { Reconhecer os comportamentos emocionais e físicos como formas de } \\
\text { comunicação não verbal. }\end{array}$ \\
\hline & & Fornecer métodos alternativos de comunicação. \\
\hline & & $\begin{array}{l}\text { Ajustar o estilo de comunicação para atender às necessidades do cliente } \\
\text { (falar na frente do paciente, escutar atentamente, apresentar uma ideia de } \\
\text { cada vez, falar devagar, utilizar comunicação escrita). }\end{array}$ \\
\hline \multirow{3}{*}{ Confusão aguda (00128) } & \multirow{3}{*}{$\begin{array}{l}\text { Estado neurológico } \\
\quad(0909) \\
\text { (Indicador: consciência) }\end{array}$} & Controle do ambiente: segurança (6486) \\
\hline & & $\begin{array}{l}\text { Identificar as necessidades de segurança do paciente com base na condição } \\
\text { física e cognitiva. }\end{array}$ \\
\hline & & Usar dispositivos de proteção para evitar situações perigosas. \\
\hline \multirow{6}{*}{$\begin{array}{l}\text { Débito cardíaco } \\
\text { diminuído (00029) }\end{array}$} & \multirow{6}{*}{$\begin{array}{l}\text { Efetividade da bomba } \\
\text { cardíaca (0400) } \\
\text { (Indicador: pressão } \\
\text { arterial sistólica) }\end{array}$} & Prevenção do choque (4260) \\
\hline & & Monitorar possíveis fontes de perda de líquidos (drenos, sondas, lesões). \\
\hline & & $\begin{array}{l}\text { Monitorar a condição circulatória (pressão arterial, cor da pele, temperatura } \\
\text { da pele, sons cardíacos, frequência e ritmo cardíaco, presença e qualidade } \\
\text { do pulso periférico, enchimento capilar). }\end{array}$ \\
\hline & & Monitorar oximetria e ECG. \\
\hline & & Inserir e manter acesso EV de grande calibre. \\
\hline & & $\begin{array}{l}\text { Administrar líquidos EV e medicações específicas conforme protocolo/ } \\
\text { prescrição médica. }\end{array}$ \\
\hline \multirow{7}{*}{$\begin{array}{l}\text { Desobstrução ineficaz das } \\
\text { vias aéreas }(00031)\end{array}$} & \multirow{7}{*}{$\begin{array}{l}\text { Estado respiratório } \\
\text { (0415) } \\
\text { (Indicador: permeabilidade } \\
\text { das vias aéreas) }\end{array}$} & Aspiração de vias aéreas (3160) \\
\hline & & $\begin{array}{l}\text { Utilizar equipamentos de proteção individual (EPI), como luvas, óculos de } \\
\text { proteção e máscara. }\end{array}$ \\
\hline & & Verificar a necessidade de aspiração oral e/ou traqueal. \\
\hline & & Auscultar os sons respiratórios antes e depois da aspiração. \\
\hline & & $\begin{array}{l}\text { Utilizar equipamento descartável esterilizado para } \\
\text { cada procedimento de aspiração. }\end{array}$ \\
\hline & & $\begin{array}{l}\text { Utilizar a menor pressão de aspiração necessária para remover secreções } \\
\text { (80 a } 120 \mathrm{mmHg} \text { para adultos). }\end{array}$ \\
\hline & & Monitorar o nível de consciência e reflexo de tosse. \\
\hline
\end{tabular}


Quadro 1. Continuação.

\begin{tabular}{|c|c|c|}
\hline $\begin{array}{l}\text { Diagnósticos de } \\
\text { enfermagem (NANDA-I) }\end{array}$ & $\begin{array}{l}\text { Resultados de } \\
\text { enfermagem } \\
\text { (NOC) }\end{array}$ & Intervenções de enfermagem (NIC) \\
\hline \multirow{10}{*}{ Dor aguda (00132) } & \multirow{10}{*}{$\begin{array}{l}\text { Satisfação do cliente: } \\
\text { controle da dor (3016) } \\
\text { (Indicador: dor } \\
\text { controlada) }\end{array}$} & Controle da dor (1400) \\
\hline & & Assegurar cuidados analgésicos para o paciente. \\
\hline & & Explorar com o paciente os fatores que melhoram/pioram a dor. \\
\hline & & $\begin{array}{l}\text { Avaliar alterações da dor para identificar potenciais agravos ao paciente } \\
\text { (frequência, intensidade/severidade, duração etc.). }\end{array}$ \\
\hline & & Orientar sobre os princípios de manejo da dor. \\
\hline & & $\begin{array}{l}\text { Observar, registrar e comunicar indicações não verbais de dor (expressão } \\
\text { facial, gestos protetores, taquicardia, sudorese, hipertensão). }\end{array}$ \\
\hline & & Manter alinhamento corporal. \\
\hline & & Investigar alergia medicamentosa antes da administração do fármaco. \\
\hline & & Verificar, registrar e comunicar alterações nos sinais vitais. \\
\hline & & $\begin{array}{l}\text { Aplicar a escala de nível de dor: } \\
\text { ( ) Sem dor: } 0 \text { a 2; } \\
\text { ( ) Dor moderada: } 3 \text { a } 7 \text {; } \\
\text { ( ) Dor intensa: } 8 \text { a } 10 \text {. }\end{array}$ \\
\hline \multirow{6}{*}{$\begin{array}{l}\text { Integridade tissular } \\
\text { prejudicada }(00044)\end{array}$} & \multirow{6}{*}{$\begin{array}{l}\text { Integridade tissular: } \\
\text { pele e mucosas ( } 1101) \\
\text { (Indicador: integridade } \\
\text { tecidual) }\end{array}$} & Cuidados com o local da incisão (3440) \\
\hline & & $\begin{array}{l}\text { Inspecionar o local da incisão para detectar alterações (sangramento, } \\
\text { vermelhidão, edema, evisceração etc.). }\end{array}$ \\
\hline & & Manter o correto posicionamento de drenos e/ou sondas. \\
\hline & & Realizar troca do curativo quando necessário. \\
\hline & & $\begin{array}{l}\text { Realizar e registrar tricotomia da pele, observando } \\
\text { indicação e área a ser operada. }\end{array}$ \\
\hline & & $\begin{array}{l}\text { Realizar e registrar degermação do campo operatório, observando as } \\
\text { características da área a ser operada e utilizando soluções antissépticas } \\
\text { padronizadas. }\end{array}$ \\
\hline \multirow{4}{*}{ Medo (00148) } & \multirow{4}{*}{$\begin{array}{l}\text { Nível de medo (1210) } \\
\text { (Indicador: medo } \\
\text { verbalizado) }\end{array}$} & Apoio emocional (5270) \\
\hline & & Fazer declarações compreensivas ou empáticas. \\
\hline & & Apoiar o uso de mecanismos de defesa apropriados. \\
\hline & & $\begin{array}{l}\text { Ficar com o paciente e proporcionar a garantia da segurança } \\
\text { e proteção durante o período. }\end{array}$ \\
\hline \multirow{5}{*}{ Náusea (00134) } & \multirow{5}{*}{$\begin{array}{l}\text { Nivel de desconforto } \\
\text { (2109) (Indicador: } \\
\text { náusea) }\end{array}$} & Controle da náusea (1450) \\
\hline & & Observar sinais não verbais de desconforto. \\
\hline & & Identificar fatores que possam causar a náusea ou contribuir com ela. \\
\hline & & Certificar-se da efetividade dos medicamentos antieméticos administrados. \\
\hline & & $\begin{array}{l}\text { Observar e registrar frequência, duração e intensidade } \\
\text { dos episódios de náuseas. }\end{array}$ \\
\hline \multirow{5}{*}{$\begin{array}{l}\text { Padrão respiratório } \\
\text { ineficaz (00032) }\end{array}$} & \multirow{5}{*}{$\begin{array}{l}\text { Estado respiratório } \\
(\mathbf{0 4 1 5 )} \\
\text { (Indicador: frequência, } \\
\text { ritmo e profundidade } \\
\text { respiratória) }\end{array}$} & Monitoração respiratória (3350) \\
\hline & & Monitorar frequência, ritmo, profundidade e esforço das respirações. \\
\hline & & $\begin{array}{l}\text { Observar os movimentos torácicos, notando assimetria, uso dos músculos } \\
\text { acessórios e retração da musculatura supraclavicular e intercostal. }\end{array}$ \\
\hline & & Monitorar os níveis de saturação de $\mathrm{O}_{2}$. \\
\hline & & $\begin{array}{l}\text { Abrir vias aéreas utilizando a técnica da elevação do } \\
\text { queixo ou tração da mandíbula. }\end{array}$ \\
\hline
\end{tabular}


Quadro 1. Continuação.

\begin{tabular}{|c|c|c|}
\hline $\begin{array}{l}\text { Diagnósticos de } \\
\text { enfermagem (NANDA-I) }\end{array}$ & $\begin{array}{l}\text { Resultados de } \\
\text { enfermagem } \\
\text { (NOC) }\end{array}$ & Intervenções de enfermagem (NIC) \\
\hline \multirow{7}{*}{ Risco de aspiração (00039) } & \multirow{7}{*}{$\begin{array}{l}\text { Prevenção da } \\
\text { aspiração (1918) } \\
\text { (Indicador: identifica } \\
\text { fatores de risco) }\end{array}$} & Precauções contra aspiração (3200) \\
\hline & & $\begin{array}{l}\text { Monitorar o nível de consciência, reflexo de tosse, } \\
\text { reflexo de vômito e capacidade de deglutição. }\end{array}$ \\
\hline & & Manter via aérea. \\
\hline & & Manter o balonete traqueal insuflado, conforme apropriado. \\
\hline & & Usar agentes procinéticos, se adequado. \\
\hline & & $\begin{array}{l}\text { Manter sistema de } \mathrm{O}_{2} \text { e aspiração das vias aéreas } \\
\text { acessíveis para uso imediato. }\end{array}$ \\
\hline & & $\begin{array}{l}\text { Aspirar vias aéreas, registrar e comunicar características } \\
\text { e quantidade das secreções. }\end{array}$ \\
\hline \multirow{3}{*}{$\begin{array}{l}\text { Risco de contaminação } \\
(00180)\end{array}$} & \multirow{3}{*}{$\begin{array}{l}\text { Controle de riscos } \\
\quad(1902) \\
\text { (Indicador: identifica os } \\
\text { fatores de risco) }\end{array}$} & Proteção contra riscos ambientais (8880) \\
\hline & & Avaliar o ambiente em relação a riscos reais e potenciais. \\
\hline & & $\begin{array}{l}\text { Monitorar a incidência de doenças e lesões relacionadas } \\
\text { aos perigos ambientais. }\end{array}$ \\
\hline \multirow{6}{*}{$\begin{array}{l}\text { Risco de dignidade humana } \\
\text { comprometida (00174) }\end{array}$} & \multirow{6}{*}{$\begin{array}{c}\text { Satisfação do cliente: } \\
\text { proteção dos direitos } \\
\text { (3008) } \\
\text { (Indicador: manutenção } \\
\text { da privacidade) }\end{array}$} & Proteção dos direitos do paciente $(\mathbf{7 4 6 0})$ \\
\hline & & Garantir a privacidade do paciente durante os procedimentos. \\
\hline & & Verificar necessidades especiais e individuais do paciente. \\
\hline & & Observar a preferência religiosa. \\
\hline & & Intervir em situações que envolvam cuidado inseguro e inadequado. \\
\hline & & Manter a confidencialidade com a informação de saúde do paciente. \\
\hline \multirow{10}{*}{$\begin{array}{l}\text { Risco de disfunção } \\
\text { neurovascular } \\
\text { periférica (00086) }\end{array}$} & \multirow{10}{*}{$\begin{array}{c}\text { Consequências da } \\
\text { imobilidade fisiológica } \\
\text { (0204) } \\
\text { (Indicadores: estase } \\
\text { venosa, força muscular, } \\
\text { movimento articular) }\end{array}$} & Posicionamento: intraoperatório (0842) \\
\hline & & Determinar a amplitude de movimento e a estabilidade articular. \\
\hline & & Checar a circulação periférica e o estado neurológico. \\
\hline & & $\begin{array}{l}\text { Coordenar a transferência e o posicionamento com o estágio da anestesia } \\
\text { ou o nível de consciência. }\end{array}$ \\
\hline & & Utilizar equipamentos auxiliares para suportar extremidades e cabeça. \\
\hline & & Manter o alinhamento corporal do paciente. \\
\hline & & Fazer uso de dispositivos para prevenir a formação de trombos venosos. \\
\hline & & $\begin{array}{l}\text { Avaliar, registrar e comunicar alterações da pele (coloração, edema, } \\
\text { textura, calor, ulceração) no local de aplicação do torniquete. }\end{array}$ \\
\hline & & Comunicar o cirurgião sobre o tempo do torniquete a intervalos regulares. \\
\hline & & $\begin{array}{l}\text { Avaliar, registrar e comunicar alterações da pele sob o manguito após } \\
\text { remoção, além de avaliar pulso periférico, sensibilidade/capacidade para } \\
\text { movimentar os dedos após desinflar o manguito. }\end{array}$ \\
\hline \multirow{4}{*}{$\begin{array}{l}\text { Risco de glicemia } \\
\text { instável (00179) }\end{array}$} & \multirow{4}{*}{$\begin{array}{l}\text { Nível de gicose no } \\
\text { sangue ( } 2300) \\
\text { (Indicador: glicose } \\
\text { do sangue) }\end{array}$} & Identificação de risco (6610) \\
\hline & & $\begin{array}{l}\text { Rever a história de saúde e documentos pregressos quanto a evidências de } \\
\text { diagnósticos e tratamentos médicos e de enfermagem prévios. }\end{array}$ \\
\hline & & Monitorar glicemia. \\
\hline & & Monitorar sinais de hiper/hipoglicemia \\
\hline
\end{tabular}


Quadro 1. Continuação.

\begin{tabular}{|c|c|c|}
\hline $\begin{array}{l}\text { Diagnósticos de } \\
\text { enfermagem (NANDA-I) }\end{array}$ & $\begin{array}{l}\text { Resultados de } \\
\text { enfermagem } \\
\text { (NOC) }\end{array}$ & Intervenções de enfermagem (NIC) \\
\hline \multirow{9}{*}{$\begin{array}{l}\text { Risco de hipotermia } \\
\text { perioperatória (00254) }\end{array}$} & \multirow{9}{*}{$\begin{array}{c}\text { Termorregulação } \\
\mathbf{( 0 8 0 0 )} \\
\text { (Indicador: hipotermia) }\end{array}$} & Regulação da temperatura: perioperatória (3902) \\
\hline & & Instituir dispositivo de monitoramento contínuo da temperatura central. \\
\hline & & $\begin{array}{l}\text { Monitorar, registrar e comunicar sinais e sintomas de } \\
\text { hipotermia ou hipertermia. }\end{array}$ \\
\hline & & Controlar temperatura corporal antes do início da cirurgia. \\
\hline & & Ajustar a temperatura do ambiente às necessidades do paciente. \\
\hline & & $\begin{array}{l}\text { Minimizar a exposição do paciente durante o preparo cirúrgico e o } \\
\text { procedimento propriamente dito. }\end{array}$ \\
\hline & & Instalar e regular dispositivo de aquecimento ativo. \\
\hline & & Monitorar temperatura ambiente. \\
\hline & & Verificar, registrar e comunicar alterações nos sinais vitais. \\
\hline \multirow{14}{*}{ Risco de infecção (00004) } & \multirow{14}{*}{$\begin{array}{l}\text { Controle de riscos: } \\
\text { processo infeccioso } \\
\qquad(1924) \\
\text { (Indicador: adoção de } \\
\text { medidas imediatas para } \\
\text { reduzir o risco) }\end{array}$} & Controle de infecção: transoperatória (6545) \\
\hline & & Monitorar e manter a temperatura da sala entre 20 e $24^{\circ} \mathrm{C}$. \\
\hline & & Monitorar e manter a umidade relativa da sala entre $20 \%$ e $60 \%$. \\
\hline & & Limitar e controlar o tráfego. \\
\hline & & $\begin{array}{l}\text { Verificar se os antibióticos profiláticos estão sendo } \\
\text { administrados apropriadamente. }\end{array}$ \\
\hline & & Usar precauções universais. \\
\hline & & Controlar o uso adequado das roupas privativas. \\
\hline & & Verificar a integridade da embalagem estéril. \\
\hline & & Verificar os indicadores de esterilização. \\
\hline & & Abrir os suprimentos e instrumentos usando técnica asséptica. \\
\hline & & Auxiliar na paramentação dos membros da equipe. \\
\hline & & $\begin{array}{l}\text { Monitorar o campo estéril para detectar quebra da } \\
\text { esterilidade e corrigir erros. }\end{array}$ \\
\hline & & Aplicar solução antimicrobiana ao local da cirurgia. \\
\hline & & $\begin{array}{l}\text { Conferir o número de compressas cirúrgicas e gazes usadas } \\
\text { nas cirurgias e cavidades. }\end{array}$ \\
\hline \multirow{10}{*}{$\begin{array}{l}\text { Risco de lesão por } \\
\text { posicionamento } \\
\text { perioperatório (00087) }\end{array}$} & \multirow{10}{*}{$\begin{array}{l}\text { Preparo pré- } \\
\text { procedimento (1921) } \\
\text { (Indicador: } \\
\text { conhecimento dos } \\
\text { riscos e complicações } \\
\text { potenciais) }\end{array}$} & Posicionamento: intraoperatório (0842) \\
\hline & & Determinar a amplitude de movimento e a estabilidade articular. \\
\hline & & Utilizar equipamentos auxiliares para imobilização. \\
\hline & & Aplicar acolchoamento sobre as proeminências ósseas. \\
\hline & & Manter o alinhamento corporal do paciente. \\
\hline & & Monitorar a posição do paciente durante a cirurgia. \\
\hline & & Registrar a posição e os equipamentos utilizados. \\
\hline & & Posicionar o paciente de forma a favorecer a ventilação/perfusão. \\
\hline & & $\begin{array}{l}\text { Avaliar, registrar e comunicar alterações da pele (coloração, edema, } \\
\text { textura, calor, lesão). }\end{array}$ \\
\hline & & $\begin{array}{l}\text { Aplicação da Escala ELPO (Escala de Avaliação de Risco para } \\
\text { o Desenvolvimento de Lesões Decorrentes do } \\
\text { Posicionamento Cirúrgico do Paciente)* } \\
\text { ( ) Menor risco: } 7 \text { a 19; } \\
\text { ( ) Maior risco: } 20 \text { a } 35 \text {. }\end{array}$ \\
\hline
\end{tabular}


Quadro 1. Continuação.

\begin{tabular}{|c|c|c|}
\hline $\begin{array}{l}\text { Diagnósticos de } \\
\text { enfermagem (NANDA-I) }\end{array}$ & $\begin{array}{l}\text { Resultados de } \\
\text { enfermagem } \\
\text { (NOC) }\end{array}$ & Intervenções de enfermagem (NIC) \\
\hline \multirow{5}{*}{$\begin{array}{l}\text { Risco de lesão na } \\
\text { córnea (00245) }\end{array}$} & \multirow{5}{*}{$\begin{array}{l}\text { Integridade tissular: } \\
\text { pele e mucosas ( } 1101 \text { ) } \\
\text { (Indicador: lesões } \\
\text { nas mucosas) }\end{array}$} & Cuidado ocular (1650) \\
\hline & & Retirar lentes de contato. \\
\hline & & Aplicar protetor ocular. \\
\hline & & Aplicar pomada lubrificante. \\
\hline & & Manter as pálpebras fechadas com fita adesiva. \\
\hline \multirow{8}{*}{$\begin{array}{l}\text { Risco de lesão térmica } \\
(00220)\end{array}$} & \multirow{8}{*}{$\begin{array}{l}\text { Integridade tissular: } \\
\text { pele e mucosas (1101) } \\
\text { (Indicador: integridade } \\
\text { tecidual) }\end{array}$} & Precauções cirúrgicas (2920) \\
\hline & & Providenciar unidade eletrocirúrgica, placa neutra e eletrodo ativo. \\
\hline & & Confirmar o funcionamento adequado da unidade eletrocirúrgica. \\
\hline & & Confirmar se o paciente não está em contato com peças metálicas. \\
\hline & & $\begin{array}{l}\text { Confirmar a ausência de marca-passo cardíaco, outros } \\
\text { implantes elétricos ou próteses metálicas que contraindiquem } \\
\text { o uso de cauterização eletrocirúrgica. }\end{array}$ \\
\hline & & $\begin{array}{l}\text { Colocar a placa neutra na pele seca e intacta, com o mínimo } \\
\text { possível de pelos, sobre ampla massa muscular e o mais } \\
\text { próximo possível do sítio operatório. }\end{array}$ \\
\hline & & $\begin{array}{l}\text { Remover agentes preparatórios inflamáveis } \\
\text { residuais antes de iniciar a cirurgia. }\end{array}$ \\
\hline & & Usar dispositivo para armazenar o eletrodo ativo durante a cirurgia. \\
\hline \multirow{3}{*}{$\begin{array}{l}\text { Risco de mucosa oral } \\
\text { prejudicada (00247) }\end{array}$} & \multirow{3}{*}{$\begin{array}{l}\text { Integridade tissular: } \\
\text { pele e mucosas (1101) } \\
\text { (Indicador: lesões } \\
\text { nas mucosas) }\end{array}$} & Inserção e estabilização de vias aéreas artificiais (3120) \\
\hline & & $\begin{array}{l}\text { Marcar o tubo endotraqueal na posição dos lábios ou narinas, usando as } \\
\text { marcações de centímetros no TE, fixar a via aérea artificial (orofaríngea ou } \\
\text { nasofaríngea) em local adequado com fita adesiva e documentar.. }\end{array}$ \\
\hline & & Monitorar mucosa oral. \\
\hline \multirow{6}{*}{ Risco de quedas (00155) } & \multirow{6}{*}{$\begin{array}{l}\text { Ambiente de cuidado à } \\
\text { saúde seguro (1943) } \\
\text { (Indicador: política de } \\
\text { prevenção de quedas) }\end{array}$} & Posicionamento: intraoperatório (0842) \\
\hline & & Travar as rodas da maca e da mesa cirúrgica. \\
\hline & & Usar número adequado de pessoas para transferir o paciente. \\
\hline & & Aplicar faixa de segurança e restrição de membros superiores. \\
\hline & & Monitorar a posição do paciente durante a cirurgia. \\
\hline & & $\begin{array}{l}\text { Aplicar escala de queda de Morse }{ }^{* *} \\
\text { ( ) Risco baixo: } 0 \text { a } 24 \text {; } \\
\text { ( ) Risco médio: } 25 \text { a } 44 \text {; } \\
\text { ( ) Risco alto >=45. }\end{array}$ \\
\hline \multirow{3}{*}{$\begin{array}{l}\text { Risco de resposta alérgica ao } \\
\text { látex }(00042)\end{array}$} & \multirow{3}{*}{$\begin{array}{l}\text { Resposta imune de } \\
\text { hipersensibilidade } \\
\text { (0707) } \\
\text { (Indicador: reações } \\
\text { alérgicas) }\end{array}$} & Precauções no uso de artigos de látex (6570) \\
\hline & & $\begin{array}{l}\text { Perguntar ao paciente ou a outra pessoa apropriada sobre a história de } \\
\text { reação sistêmica ao látex de borracha natural (edema facial ou escleral, } \\
\text { olhos lacrimejantes, urticária, rinite, chiado no peito). }\end{array}$ \\
\hline & & Monitorar os sinais e sintomas do paciente de uma reação sistêmica. \\
\hline \multirow{5}{*}{$\begin{array}{l}\text { Risco de sangramento } \\
(00206)\end{array}$} & \multirow{5}{*}{$\begin{array}{l}\text { Gravidade da perda } \\
\text { de sangue (0413) } \\
\text { (Indicador: perda visível } \\
\text { de sangue) }\end{array}$} & Precauções contra sangramento (4010) \\
\hline & & Monitorar a ocorrência de sinais e sintomas de sangramento persistente. \\
\hline & & Verificar a suspensão do uso de substâncias anticoagulantes. \\
\hline & & Administrar hemocomponentes ou hemoderivados. \\
\hline & & Manter acesso venoso pérvio, de grosso calibre. \\
\hline
\end{tabular}


Quadro 1. Continuação.

\begin{tabular}{|c|c|c|}
\hline $\begin{array}{l}\text { Diagnósticos de } \\
\text { enfermagem (NANDA-I) }\end{array}$ & $\begin{array}{l}\text { Resultados de } \\
\text { enfermagem } \\
\text { (NOC) }\end{array}$ & Intervenções de enfermagem (NIC) \\
\hline \multirow{6}{*}{$\begin{array}{l}\text { Risco de volume deficiente } \\
\text { de líquidos }(00028)\end{array}$} & \multirow{6}{*}{$\begin{array}{l}\text { Equilíbrio hídrico } \\
\text { (0601) } \\
\text { (Indicador: pressão } \\
\text { arterial) }\end{array}$} & Controle da hipovolemia (4180) \\
\hline & & Monitorar possíveis fontes de perda de líquidos. \\
\hline & & $\begin{array}{l}\text { Monitorar condição circulatória (pressão arterial, cor da pele, } \\
\text { temperatura, frequência e ritmo cardíaco, presença e qualidade } \\
\text { de pulsos periféricos e preenchimento capilar). }\end{array}$ \\
\hline & & Monitorar ECG. \\
\hline & & Inserir e manter cateter venoso de grande calibre. \\
\hline & & $\begin{array}{l}\text { Observar, comunicar e registrar a intensidade e } \\
\text { frequência das náuseas/êmese. }\end{array}$ \\
\hline \multirow{7}{*}{$\begin{array}{l}\text { Ventilação espontânea } \\
\text { prejudicada (00033) }\end{array}$} & \multirow{7}{*}{$\begin{array}{l}\text { Recuperação cirúrgica: } \\
\text { pós-operatório } \\
\text { imediato (2305) } \\
\text { (Indicador: saturação } \\
\text { de } \mathrm{O}_{2} \text { ) }\end{array}$} & Assistência ventilatória (3390) \\
\hline & & Manter via aérea pérvia. \\
\hline & & Posicionar paciente para aliviar dispneia. \\
\hline & & Encorajar respiração profunda e lenta, mudança de posição e tosse. \\
\hline & & Monitorar sinais de fadiga da musculatura respiratória. \\
\hline & & Iniciar/manter oxigenoterapia prescrita. \\
\hline & & Monitorar o estado respiratório e de oxigenação. \\
\hline
\end{tabular}

NANDA-I: North American Nursing Diagnosis Association Internacional; NOC: Classificação dos Resultados de Enfermagem; NIC: Classificação das Intervenções de Enfermagem; ECG: Eletrocardiograma; EV: Endovenoso; TE: Tubo Endotraqueal. *a validação da escala de Morse pode ser feita pelo link: $<$ http://www.scielo.br/pdf/reeusp/v47n3/0080-6234-reeusp-47-3-00569.pdf>; **a validação da escala ELPO pode ser feita pelo link: <http://www.scielo.br/pdf/rlae/v24/pt_0104-1169-rlae-24-02704.pdf>.

clínicas acuradas favorecem a prescrição de cuidados adequados, que, por sua vez, promovem a obtenção de resultados de saúde desejáveis ${ }^{12}$. Diante disso, a condução da discussão dos resultados deu-se com foco na representatividade dos diagnósticos de enfermagem elencados para o paciente que vivenciava o momento transoperatório.

Conforme a classificação NANDA-I, os 27 diagnósticos apresentados no estudo estão contidos em sete dos 13 domínios da referida classificação. São eles: segurança/proteção $(\mathrm{n}=14 ; 51,9 \%)$, atividade/repouso $(\mathrm{n}=4 ; 14,8 \%)$, nutrição $(n=2 ; 7,4 \%)$, percepção/ cognição $(n=2 ; 7,4 \%)$, enfrentamento/tolerância ao estresse $(n=2 ; 7,4 \%)$, conforto $(n=2$; $7,4 \%)$ e autopercepção ( $n=1 ; 3,7 \%)$.

É relevante destacar que grande parte dos diagnósticos elencados pertencia ao domínio "segurança/proteção", e, destes, a maioria foi classificada como "diagnósticos de risco" $(\mathrm{n}=12 ; 85,7 \%)$.

Os diagnósticos de risco estão relacionados com a vulnerabilidade do indivíduo em desenvolver uma resposta indesejável diante da sua condição clínica ${ }^{3}$. Com base nessa definição, é possível entender por que quase $60 \%$ ( $\mathrm{n}=84 ; 59,6 \%$ ) das atividades de enfermagem propostas para assistir o paciente no momento transoperatório estão voltadas para promover a sua segurança e evitar que diagnósticos de risco se tornem diagnósticos reais. Mesmo reconhecendo sua finalidade terapêutica, é inegável que todo procedimento anestésico-cirúrgico consiste em potencial dano à saúde do paciente.

Para identificar diagnósticos de enfermagem com altos graus de acurácia, o enfermeiro deve realizar um levantamento minucioso das condições clínicas e emocionais do paciente cirúrgico, das condições ambientais, da paramentação dos profissionais, do tipo de anestesia, da técnica cirúrgica a ser utilizada, da habilidade técnica da equipe de saúde, da circulação de pessoas na sala de cirurgia, da condição dos materiais cirúrgicos e das ações a serem tomadas perante os riscos inerentes ao procedimento ${ }^{13}$.

$\mathrm{Na}$ atualidade, é fundamental a priorização de ações que garantam a segurança do paciente e minimizem a ocorrência de eventos adversos associados à assistência à saúde ${ }^{14}$. A segurança do paciente está diretamente ligada ao processo de trabalho institucional, em que a falta de protocolos assistenciais ou o inadequado planejamento do cuidado podem aumentar os riscos de danos à saúde dos pacientes ${ }^{15}$. Ações resolutivas dos enfermeiros que atuam em CC contribuem para a manutenção da segurança do paciente e refletem na adequada recuperação pós-cirúrgica ${ }^{16,17}$. 
O material teórico construído favorece a realização de uma assistência de enfermagem com fundamentação científica e condizente com o perfil do paciente no período transoperatório. Nesse sentido, a maior contribuição do estudo é o esforço em consolidar o conhecimento sobre a assistência de enfermagem a ser realizada durante o período transoperatório. Como limitação da investigação, aponta-se o fato de a pesquisa ter sido desenhada com o intuito de atender às necessidades do paciente que vivencia a experiência cirúrgica sem considerar a especialidade da intervenção, faixa etária ou sexo do paciente como variáveis. Não levar em conta as particularidades de determinada clientela pode tornar o estudo demasiadamente amplo e pouco específico.

\section{CONCLUSÃO}

A versão final do conteúdo teórico construído ficou composta de 27 diagnósticos, 25 resultados, 26 intervenções e 141 atividades de enfermagem.

Acredita-se que os resultados apresentados possam ser utilizados para fundamentar a prática clínica dos enfermeiros que atuam em CC, no entanto é importante destacar que as ligações propostas aqui não devem substituir o julgamento clínico do enfermeiro perioperatório.

Ressalta-se a necessidade de novos estudos que explorem a aplicação prática das ligações propostas em diferentes cenários e em populações específicas.

\section{REFERÊNCIAS}

1. Carvalho EC, Cruz DALM, Herdman TH. Contribuição das linguagens padronizadas para a produção do conhecimento, raciocínio clínico e prática clínica da Enfermagem. Rev Bras Enferm. 2013; 66(esp):13441. http://dx.doi.org/10.1590/S0034-71672013000700017

2. Chaves IC, Jacoby LC, Cecchetto FH. Diagnósticos de enfermagem mais frequentes aos pacientes submetidos à cirurgia cardíaca nos períodos pré, trans e pós-operatórios. Rev Cuidado Enf Cesuca [Internet]. 2016[citado 14 fev. 2018];2(2):1-15. Disponível em: http://ojs.cesuca.edu.br/index.php/revistaenfermagem/ article/view/953

3. Herdman TH, Shiguemi K. Diagnósticos de enfermagem da NANDA: definições e classificações 2015-2017. Porto Alegre: Artmed; 2015.

4. Bulechek GM, Butcher HK, Dochterman J, Wagner CM. Classificação das intervenções de enfermagem - NIC. 6ª ed. São Paulo: Elsevier; 2016.

5. Johnson M, Moorhead S, Maas ML, Swanson E. Classificação dos resultados de enfermagem - NOC. 5a ed. São Paulo: Elsevier; 2016.

6. Jonhson M, Moorhead S, Bulechek G, Butcher H, Maas M, Swanson E. Ligações NANDA-NOC-NIC: condições clínicas: suporte ao raciocínio e assistência de qualidade. Rio de Janeiro: Elsevier; 2012.

7. Castellanos BEP, Jouclas VMG. Assistência de enfermagem perioperatória: um modelo conceitual. Rev Esc Enferm USP. 1990;24(3):359-70.

8. Lemos CS, Suriano MLF. Desenvolvimento de um instrumento: metodologia de ensino para aprimoramento da prática perioperatória. Rev SOBECC. 2013;18(4):57-69.

9. Sena AC, Nascimento ERP, Maia ARCR, Santos JLG. Collective preparation of a nursing care instrument for immediate preoperative patients. Rev Baiana Enf [Internet]. 2017[citado 14 fev. 2018];31(1):e20506. Disponível em: https://portalseer.ufba. br/index.php/enfermagem/article/view/20506/14111 http://dx.doi. org/10.18471/rbe.v31i1.20506
10. Lemos CS, Poveda VB, Peniche ACG. Construction and validation of a nursing care protocol in anesthesia. Rev Latino-Am Enferm. 2017;25:e2952. http://dx.doi.org/10.1590/1518-8345.2143.2952

11. Alexandre NMC, Coluci MZO. Validade de conteúdo nos processos de construção e adaptação de instrumentos de medidas. Ciênc Saúde Coletiva [Internet]. 2011 [citado 14 fev. 2018];16(7):3061-8. Disponível em: http://www.scielo.br/pdf/csc/v16n7/06.pdf

12. Matos FGOA, Cruz DALM. Development of an instrument to evaluate diagnosis accuracy. Rev Esc Enferm USP. 2009;43(Esp):1088-97. http://dx.doi.org/10.1590/S0080-62342009000500013

13. Botarelli FR, Queiroz QJR, Fernandes APNL, Araújo JNM, Ferreira Júnior MA, Vitor AF. Diagnóstico de enfermagem risco de infecção em pacientes no pós-operatório: estudo transversal. Online Braz J Nurs [Internet]. 2016[citado 14 fev. 2018];15(2):245-53. Disponível em: http://www.objnursing.uff.br/index.php/nursing/article/view/5299 http://dx.doi.org/10.17665/1676-4285.20165299

14. Oliveira MM, Mendonça KM. Análise da visita pré-operatória de enfermagem: revisão integrativa. Rev SOBECC. 2014;19(3):164-72.

15. Reis GAX, Valera IMA, Inoue KC, Souza VS, Costa MAR, Matsuda LM. Implantação das estratégias de segurança do paciente: percepções de enfermeiros gestores. Vigil Sanit Debate [Internet]. 2016[citado 15 fev. 2018];4(4):132-8. Disponivel em: https://visaemdebate.incqs. fiocruz.br/index.php/visaemdebate/article/view/801/350 http://dx.doi. org/10.22239/2317-269X.00801

16. Dalcól C, Garanhani ML. Management role of surgical center nurses: perceptions by means of images. Rev Eletr Enf [Internet]. 2016[citado 15 fev. 2018]; 18:e1168. Disponível em: https://revistas.ufg.br/fen/ article/view/34888 https://doi.org/10.5216/ree.v18.34888

17. Henriques AHB, Costa SS, Lacerda JS. Nursing care in surgical patient safety: an integrative review. Cogitare Enferm [Internet]. 2016[citado 15 fev. 2018];21(4):1-8. Disponível em: http://revistas. ufpr.br/cogitare/article/view/45622/pdf_en 